\title{
Hoyle state and rotational features in Carbon-12 within a no-core shell model framework
}

\author{
Alison C. Dreyfuss \\ Keene State College, Keene, New Hampshire 03435, USA
}

\begin{abstract}
Kristina D. Launey, Tomáš Dytrych, and Jerry P. Draayer
Department of Physics and Astronomy, Louisiana State University, Baton Rouge, LA 70803, USA

Chairul Bahri

Department of Physics, University of Notre Dame, Notre Dame, Indiana 46556-5670, USA
\end{abstract}

\begin{abstract}
By using only a fraction of the model space extended beyond current no-core shell-model limits and a many-nucleon interaction with a single parameter, we gain additional insight within a symmetryguided shell-model framework, into the many-body dynamics that gives rise to the ground state rotational band together with phenomena tied to alpha-clustering substructures in the low-lying states in ${ }^{12} \mathrm{C}$, and in particular, the challenging Hoyle state and its first $2^{+}$and $4^{+}$excitations. For these states, we offer a novel perspective emerging out of no-core shell-model considerations, including a discussion of associated nuclear deformation and matter radii. This, in turn, provides guidance for $a b$ initio shell models by informing key features of nuclear structure and the interaction.
\end{abstract}

Our present-day knowledge of various phenomena of astrophysical significance, such as nucleosynthesis, the evolution of primordial stars in the Universe, and X-ray bursts depends on reaction rates for the stellar triple$\alpha$ process, which can considerably affect, e.g., results of core-collapse supernovae simulations and stellar evolution models, predictions regarding X-ray bursts, as well as estimates of carbon production in asymptotic giant branch (AGB) stars [1]. These rates, in turn, are greatly influenced by accurate measurements and theoretical predictions of several important low-lying states in ${ }^{12} \mathrm{C}$, including the second $\mathrm{O}_{2}^{+}$(Hoyle) state and its $2^{+}$ excitation that continues to foster debate in experimental studies [2 17. Further challenges relate to the longrecognized alpha-cluster substructure of these states that has been explored within cluster-tailored [8, 9] or selfconsistent 10 microscopic framework, but has hitherto precluded an accurate - from first principles (ab initio) - no core shell-model (NCSM) description [11. Only recently, first $a b$ initio state-of-the-art calculations have been attempted using lattice effective field theory (EFT) [12.

In this letter, we report on a first study of these phenomena in ${ }^{12} \mathrm{C}$ within a no-core shell-model framework with essentially no limitation on the number of harmonic oscillator (HO) shells included in the model space. While such model spaces remain inaccessible by $a b$ initio shell models, we are able to address a long-standing challenge [13], namely, understanding highly-deformed spatial configurations from a shell-model perspective. This is achieved by down-selecting, first, to the most physically relevant nuclear configurations and, second, to pieces of the nucleon-nucleon $(N N)$ interaction that enter in commonly used nuclear potentials [14, 15].

We emphasize that the goal of the present study is to inform key features of nuclear structure and the interaction, and hence, to provide guidance needed for ab initio shell model approaches. To do so, we retain simplicity by focusing on two essential pieces, namely, the longrange part of the central nuclear force and a spin-orbit term, while excluding from the present analysis various interaction terms, such as short-range and tensor forces. The latter are indispensable for accurate descriptions, but appear to be of secondary importance to the present study, as suggested by the reasonably close agreement of the model outcome with experiment and ab initio results in smaller spaces. The outcome further points to the need for simple many-body interactions beyond twobody realistic ones for a description of large-deformation and cluster phenomena, and manifests an indication that achieving $a b$ initio descriptions is within the reach of the NCSM. The latter, in turn, will bring forward an accurate reproduction and reliable prediction of energy spectra and associated transition rates that majorly impact astrophysical studies.

In particular, this study allows one to gain further insight into the many-body dynamics, including the physically relevant deformation and particle-hole configurations, that gives rise to the ground state (g.st.) rotational band (the lowest $0^{+}, 2^{+}$and $4^{+}$states) together with low-lying states suggested to have a cluster structure $\left(0_{2}^{+}\right.$Hoyle state and its $2^{+}$and $4^{+}$excitations), as well as a third low-lying $0_{3}^{+}$state in ${ }^{12} \mathrm{C}$. We focus on excitation energies and other observables such as matter rms radii, electric quadrupole moments and E2 transition rates, as well as compare to wavefunctions obtained by ab initio shell-model calculations using a realistic $N N$ interaction. With no parameter adjustment, the present model we find is also extensible to other light nuclei, as demonstrated [16], for example, for the g.st. rotational 
band of ${ }^{8} \mathrm{Be}$ (and its low-lying $0^{+}$states) as well as of ${ }^{22} \mathrm{Ne}$ and ${ }^{22,24} \mathrm{Mg}$.

Symmetry-adapted shell-model framework. We employ the no-core symplectic model (NCSpM) for symmetry-preserving interactions with $\operatorname{Sp}(3, \mathbb{R})$ the underpinning symmetry [17. This symmetry is found inherent to nuclear dynamics - a result we have demonstrated in an analysis of large-scale $a b$ initio NCSM applications for ${ }^{12} \mathrm{C}$ and ${ }^{16} \mathrm{O}$ [18. The model offers a microscopic description of $A$ nucleons in terms of mixed deformation configurations and associated rotations [19, directly related to particle relative (with respect to the center of mass, CM) position and momentum coordinates, $\mathbf{r}_{i}$ and $\mathbf{p}_{i}$, with $i=1, \ldots, A$. It has been successfully applied to ${ }^{20} \mathrm{Ne}$ [20] with a ${ }^{16} \mathrm{O}$ core, as wells as to ${ }^{166} \mathrm{Er}$ using the Davidson potential 21]. It is a microscopic realization of the Bohr-Mottelson collective model [15, as well as a multiple HO shell generalization of Elliott's SU(3) model 14.

The NCSpM utilizes a symplectic basis (for details, see [22]), which is related - via a unitary transformation - to the three-dimensional HO ( $m$-scheme) many-body basis used in the NCSM [23. The NCSM basis is constructed using $\mathrm{HO}$ single-particle states. It is characterized by the $\hbar \Omega$ oscillator strength and by the cutoff in total excitation oscillator quanta, $N_{\max }$. Indeed, the NCSpM employed within a full model space up through $N_{\max }$, will coincide with the NCSM for the same $N_{\max }$ cutoff. It is therefore clear that the present study, while downselecting to the most relevant configurations, provides the first shell-model calculations carried beyond current NCSM limits. These important configurations are chosen among all possible symplectic $\operatorname{Sp}(3, \mathbb{R})$ irreducible representations (irreps) within the model space.

The $\operatorname{Sp}(3, \mathbb{R})$ irreps divide the space into 'vertical slices' that are comprised of basis states of definite $(\lambda \mu)$ quantum numbers of $\mathrm{SU}(3)$ (Fig. 1) linked to the intrinsic quadrupole deformation 24]. E.g., the simplest cases, $(00),(\lambda 0)$, and $(0 \mu)$, describe spherical, prolate, and oblate deformation, respectively, while a general nuclear state is typically a superposition of several hundred various triaxial deformation configurations. The basis states are built over a bandhead (Fig. 1. Set I) by consecutive $2 \hbar \Omega$ 1p-1h (1-particle-1-hole) excitations (Fig. 1. Set II), together with a smaller $2 \hbar \Omega 2 \mathrm{p}-2 \mathrm{~h}$ (two particles a shell up) correction for eliminating the spurious CM motion (not shown in the figure). In the NCSpM, to eliminate the spurious CM motion, we use symplectic generators constructed in relative coordinates with respect to the CM. These generators are used to build the basis, the interaction, the many-particle kinetic energy operator, as well as to evaluate observables.

For the purposes of this study, we utilize a microscopic many-body interaction suitable for large- $N_{\max }$ no-core shell model applications. Specifically, along with the usual spin-orbit term, we employ an elementary form tied

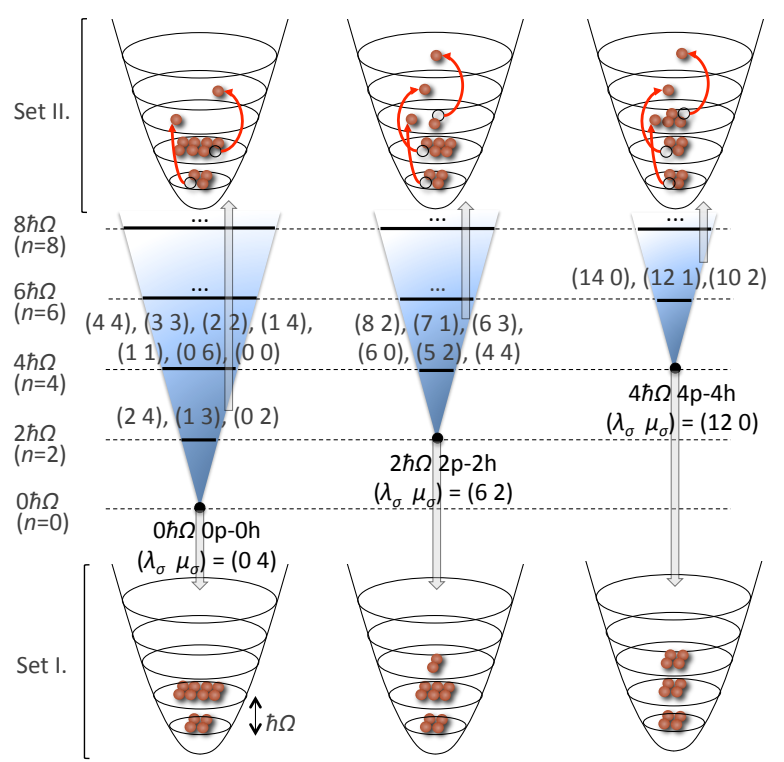

FIG. 1: $\operatorname{Sp}(3, \mathbb{R})$ irreps (slices) that comprise the spin-zero model space used for the ${ }^{12} \mathrm{C}$ NCSpM calculations. Basis states $(\lambda \mu)$ of a slice are built by $2 \hbar \Omega 1 \mathrm{p}-1 \mathrm{~h}$ monopole or quadrupole excitation (Set II) over a bandhead. The symplectic bandhead (Set I) is a $\mathrm{SU}(3)$-coupled many-body state with a given nucleon distribution over the HO shells. The corresponding $\mathrm{HO}$ energy of this nucleon configuration together with the bandhead deformation, $\left(\lambda_{\sigma} \mu_{\sigma}\right)$, serve to label the symplectic irrep.

to a long-range expansion of the nucleon-nucleon central force $V\left(\left|\mathbf{r}_{i}-\mathbf{r}_{j}\right|\right)$ 25] kept as simple as possible by considering the most relevant degrees of freedom for a description of deformed spatial configurations [14, 15,

$H_{\gamma}=\sum_{i=1}^{A}\left(\frac{\mathbf{p}_{i}^{2}}{2 m}+\frac{m \Omega^{2} \mathbf{r}_{i}^{2}}{2}\right)+\frac{\chi}{2} \frac{\left(e^{-\gamma Q \cdot Q}-1\right)}{\gamma}-\kappa \sum_{i=1}^{A} l_{i} \cdot s_{i}$.

This Hamiltonian is given in terms of particle coordinates relative to the CM, with $Q_{(2 \mathcal{M})}=\sum_{i=1}^{A} q_{(2 \mathcal{M}) i}=$ $\sum_{i} \sqrt{16 \pi / 5} r_{i}^{2} Y_{(2 \mathcal{M})}\left(\hat{\mathbf{r}}_{i}\right)$ the mass quadrupole moment and with $\frac{1}{2} Q \cdot Q=\frac{1}{2} \sum_{i} q_{i} \cdot\left(\sum_{j} q_{j}\right)$ the interaction of each particle with the total quadrupole moment of the system $^{1}$. Interactions that preserve the $\operatorname{Sp}(3, \mathbb{R})$ symmetry, such as $H_{\gamma}$ with no spin-orbit term, do not mix symplectic vertical slices, as well as greatly facilitate the use of a group-theoretical apparatus and analytical expressions for the Hamiltonian matrix elements, which, in turn, renders large $N_{\max }$ spaces manageable. Indeed, $\mathrm{Sp}(3, \mathbb{R})$ -

1 Although a technical detail, it is important to note that $Q \cdot Q$ in (1) denotes the $Q \cdot Q-\langle Q \cdot Q\rangle_{n}$ interaction, where the $\langle Q \cdot Q\rangle_{n}$ term is subtracted from $Q \cdot Q$ to eliminate a spurious shift in the zero-point energy by the average contribution, $\langle Q \cdot Q\rangle_{n}$, of $Q \cdot Q$ within the subspace of $n \mathrm{HO}$ excitations 26 27. 


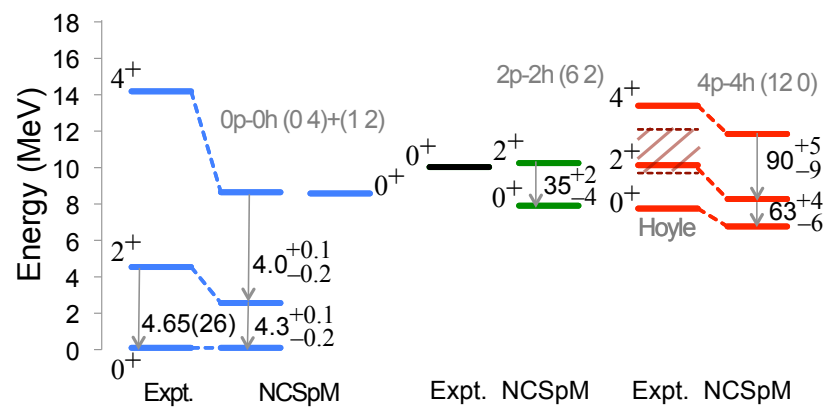

FIG. 2: NCSpM energy spectrum of ${ }^{12} \mathrm{C}$. Experimental data is from 32, except the latest results for $0_{3}^{+} 4$ and the states above the Hoyle state, $4^{+}\left[33\right.$ and $2^{+}$[7] (with a shaded area showing the energy range from $[2,6]) . B(E 2)$ transition rates are in W.u.; theoretical uncertainties are estimated for a $\pm 60 \%$ deviation of the Hoyle state energy.

symmetric Hamiltonians are particularly suitable to capture the essential characteristics of the low-energy nuclear kinematics and dynamics. The reason is that such Hamiltonians can include the many-particle kinetic energy, $\sum_{i} \mathbf{p}_{i}^{2} /(2 m)$, the HO potential, $\sum_{i} m \Omega^{2} \mathbf{r}_{i}^{2} / 2$, as well as terms dependent on $Q$ and on the orbital momentum, $\mathbf{L}=\sum_{i} \mathbf{r}_{i} \times \mathbf{p}_{i}$.

The $H_{\gamma}$ introduces simple but important many-body interactions that enter in a prescribed hierarchical way given in powers of a small positive parameter $\gamma$, the only adjustable parameter in the model. The NCSpM, with $H_{\gamma}$, reduces to the established Elliott model in the limit of a single valence shell and zero $\gamma$ and $\kappa$, where it was shown to effectively describe rotational features of light nuclei [14. A successful extension to multiple shells has been achieved and applied to the ${ }^{24} \mathrm{Mg}$ g.st. rotational band [28, where an interaction given as a polynomial in $Q$ up through $(Q \cdot Q)^{2}$ was employed. Such an interaction directly ties to our effective Hamiltonian (1). Indeed, while higher-order terms in $Q \cdot Q$ of Eq. (1) account for a renormalization of the $\chi$ coupling constant as shown in Ref. [29], they become quickly negligible for a reasonably small $\gamma$ and, e.g., for ${ }^{12} \mathrm{C}$, we find only one term (three terms), besides $Q \cdot Q$, to be sufficient for the g.st. (Hoyle state).

Collective features in ${ }^{12} \mathrm{C}$. - The low-lying energy spectrum and eigenstates for ${ }^{12} \mathrm{C}$ were calculated using the NCSpM with $H_{\gamma}$ of Eq. (1) with the empirical estimates $\hbar \Omega \approx 41 / A^{1 / 3}=18 \mathrm{MeV}$ and $\kappa \approx 20 / A^{2 / 3}=3.8$ $\mathrm{MeV}$ (e.g., see [15]). We fix the coupling constant $\chi$ by $\hbar \Omega$ and take it to decrease with increasing $N$, the total HO quanta, by using the relation derived by Rowe [30], $\chi=\frac{\hbar \Omega}{4 N}$ to a leading order. This derivation for $\chi$ is based on self-consistent arguments and is used in an $\operatorname{Sp}(3, \mathbb{R})$-based study of cluster states of ${ }^{16} \mathrm{O}[31$.

The results are shown for $N_{\max }=20$, which we found sufficient to yield convergence. This $N_{\max }$ model space is
TABLE I: NCSpM point-particle rms matter radii and electric quadrupole moments for ${ }^{12} \mathrm{C}$ compared to experimental (experimentally deduced) data. See text for a comparison to $r_{\text {rms }}$ predictions of other models.

\begin{tabular}{l|llll}
\hline & \multicolumn{2}{|c}{ matter } & $r_{\mathrm{rms}}, \mathrm{fm}$ & \multicolumn{2}{c}{$Q, e \mathrm{fm}^{2}$} \\
& Expt. & NCSpM & Expt. & NCSpM \\
\hline $0_{g s}^{+}$ & $2.43(2)^{a}$ & $2.43(1)$ & & \\
$0_{2}^{+}$(Hoyle) & $2.89(4)^{b *}$ & $2.93(5)$ & & \\
$0_{3}^{+}$ & $\mathrm{N} / \mathrm{A}$ & $2.78(4)$ & & \\
$2_{1}^{+}$ & $2.36(4)^{b *}$ & $2.42(1)$ & $+6(3)^{d}$ & $+5.9(1)$ \\
$2^{+}$above $0_{2}^{+}$ & $3.07(13)^{c *}$ & $2.93(5)$ & $\mathrm{N} / \mathrm{A}$ & $-21(1)$ \\
$4_{1}^{+}$ & $\mathrm{N} / \mathrm{A}$ & $2.41(1)$ & $\mathrm{N} / \mathrm{A}$ & $+8.0(3)$ \\
$4^{+}$above $0_{2}^{+}$ & $\mathrm{N} / \mathrm{A}$ & $2.93(5)$ & $\mathrm{N} / \mathrm{A}$ & $-26(1)$ \\
\hline
\end{tabular}

${ }^{a}$ Ref. [35; ; ${ }^{b}$ Ref. [36; ; ${ }^{c}$ Ref. [37; and ${ }^{d}$ Ref. [32].

* Experimentally deduced, based on model-dependent analyses of diffraction scattering; $0_{g s}^{+} r_{\mathrm{rms}}=2.34 \mathrm{fm}$.

further reduced by selecting the most relevant symplectic irreps of total dimensionality of $6.6 \times 10^{3}$. These irreps (vertical slices) include symplectic excitations that start at the $0 \hbar \Omega 0 \mathrm{p}-0 \mathrm{~h}(04), 2 \hbar \Omega 2 \mathrm{p}-2 \mathrm{~h}(62)$, and $4 \hbar \Omega 4 \mathrm{p}-4 \mathrm{~h}$ (120) bandheads of total spin $S=0$ (proton and neutron spins $S_{\mathrm{p}, \mathrm{n}}=0$ ) (Fig. 1), as well as two $0 \hbar \Omega$ 0p-0h (12) bandheads of $S=1$ with $S_{\mathrm{p}(\mathrm{n})}=0(1)$ and $1(0)$. In comparison to the experimental energy spectrum (Fig. 2 , the outcome reveals that, for $\gamma=1.7 \times 10^{-4}$, the $0 \mathrm{p}-$ $0 \mathrm{~h}$ and $4 \mathrm{p}-4 \mathrm{~h}$ symplectic irreps track with the rotational bands of the g.st. and Hoyle state, respectively, while the lowest $2 \mathrm{p}-2 \mathrm{~h} 0^{+}$is found to lie above the other two $0^{+}$states, close to the $10-\mathrm{MeV} 0^{+}$resonance observed in ${ }^{12} \mathrm{C}$. Clearly, we find no other $0^{+}$state lying below the calculated Hoyle state for the $\gamma$ chosen, even if all $0 \hbar \Omega$ symplectic irreps were included in the $N_{\max }=20$ model space. And further, e.g., for $2 \hbar \Omega(24), 4 \hbar \Omega(82)$, $(44)$, and (06), $6 \hbar \Omega(140)$, as well as $8 \hbar \Omega(160)$, the lowest state $\left(0^{+}\right)$lies much higher than $30 \mathrm{MeV}$. We note that the symmetry-mixing spin-orbit term is turned on only for the symplectic bandheads (up through $N_{\max }=8$ in this study). Neglecting the spin-orbit force results in a rather compressed spectrum. This is similar to the findings of early cluster models, which remedy this by allowing for alpha-cluster dissociation due to a spin-orbit force as discussed in Ref. 34.

The model with the selected $\gamma$ successfully reproduces other observables for ${ }^{12} \mathrm{C}$ that are informative of the state structure, such as $r_{\text {rms }}$ point-particle matter rms radii, $Q$ electric quadrupole moments (Table $\mathrm{I}$ ) and $B(E 2)$ transition strengths (Fig. 2). Theoretical uncertainties in this study are estimated for a $\pm 60 \%$ deviation of the Hoyle state energy. This large tolerance corresponds to only $-8 \%$ to $15 \%$ variation in $\gamma$ and, practically, has no considerable effect on these observables, e.g., it results in a $0.3-1.6 \%(2.3-10 \%)$ variation for radii ( $E 2$ transition strengths). The NCSpM finds a quite reasonable matter rms radius for the g.st. (Table I). Interestingly, our cal- 

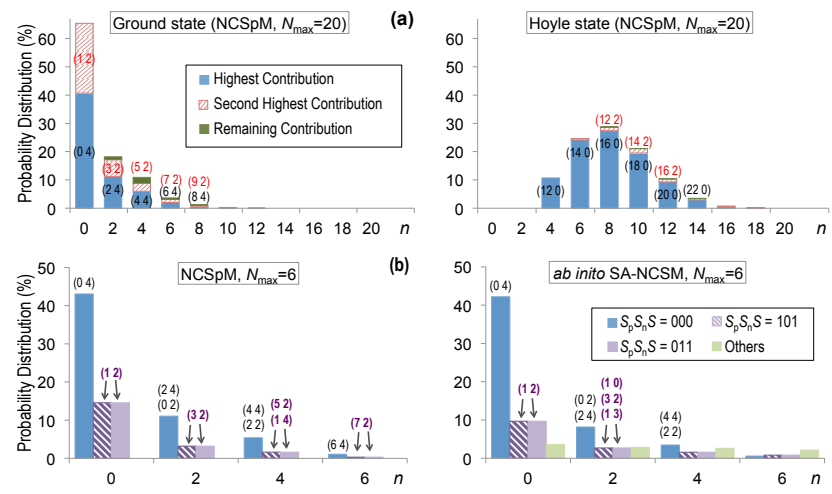

FIG. 3: Probability distribution for ${ }^{12} \mathrm{C}$ vs. the $n$ total excitations of (a) the ground state (left) and Hoyle state (right) as calculated by NCSpM in $N_{\max }=20$, and (b) the ground state as calculated by NCSpM (left) and SA-NCSM (right) for $N_{\max }=6$ and $\hbar \Omega=18 \mathrm{MeV}$. Dominant deformation modes (with probability amplitude $\geq 1 \%$ ), specified by $(\lambda \mu)$, are also shown.

culations yield matter $r_{\mathrm{rms}}=2.93 \mathrm{fm}$ for the Hoyle state, 1.2 times larger than that of the ground state. While this result drastically differs from predictions of cluster models, e.g., $3.38 \mathrm{fm}$ [8], $3.83 \mathrm{fm}[38$ and $4.31 \mathrm{fm} \mathrm{[39}$, it is close to a recent value deduced from experiment, 2.89(4) $\mathrm{fm}$ [36, as well as tracks with the ab initio lattice EFT results at a leading order, 2.4(2) fm [12.

Furthermore, the model yields a $Q_{2_{1}^{+}}$very close to the experimental value, and a large negative one for the $2^{+}$ above the Hoyle state (Table I) indicating a substantial prolate deformation for the Hoyle and $2^{+}$states. Such a deformation, albeit not so pronounced, has been also suggested by the $a b$ initio lattice EFT [12. This is also supported by the wavefunction distribution for the g.st. and Hoyle-state rotational bands (see, Fig. 3 a for the lowest $0^{+}$states). Namely, while the predominant component of the $0 \mathrm{p}-0 \mathrm{~h}$ states is at $0 \hbar \Omega(n=0)$ and manifests an evident oblate shape [as indicated by the $(\lambda \mu)=(04)$ and (12) deformation labels], the Hoyle-state band peaks around $8 \hbar \Omega(n=8)$ with a clear evidence for a prolate deformation with (160) being the largest contribution. Moreover, the Hoyle state emerges from a $4 \mathrm{p}-4 \mathrm{~h}$ shellmodel configuration, that is, the (120) bandhead (Fig. 1. set I) is realized by an alpha-particle configuration - spatially spherical (0 0), spin zero, and isospin zero - in each of the three lowest HO shells (implying spatial displacement). This together with the strong prolate deformation of this state supports an underlying alpha-particle cluster structure.

The NCSpM $B\left(E 2 ; 2^{+} \rightarrow 0^{+}\right)$estimates are also found to agree with experiment for the g.st. band, and with the value of 62.5 W.u. of Ref. 9 for the Hoyle-state band (Fig. 2). However, nonzero interband $B\left(E 2 ; 0_{2}^{+} \rightarrow\right.$ $\left.2_{1}^{+}\right)$and $M\left(E 0 ; 0_{2}^{+} \rightarrow 0_{1}^{+}\right)$strengths can only result from

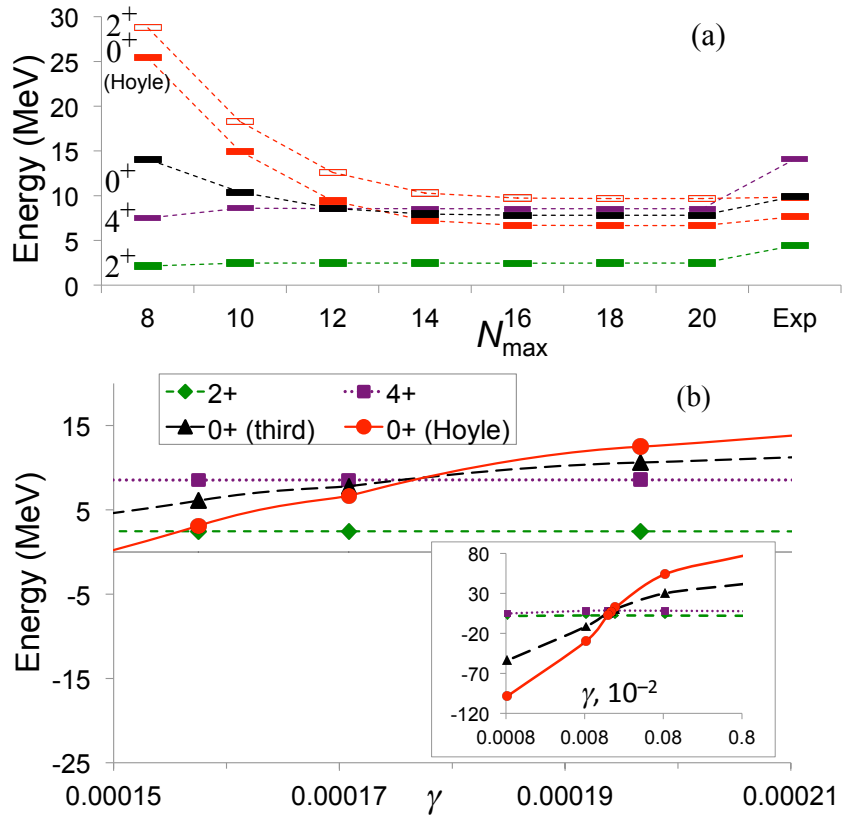

FIG. 4: Dependence of the ${ }^{12} \mathrm{C}$ NCSpM energy spectrum on (a) the model space $\left(N_{\max }\right)$ for $\gamma=1.7 \times 10^{-4}$ and (b) on the $\gamma$ parameter for $N_{\max }=20$.

mixing of $\operatorname{Sp}(3, \mathbb{R})$ irreps, which requires a symmetrybreaking interaction. But this can enter perturbatively, as less than a $2 \%$ mixing of the (120) irrep into the (04) irrep can already yield the correct order of magnitude, namely, $B\left(E 2 ; 0_{2}^{+} \rightarrow 2_{1}^{+}\right)=8.4$ W.u. and $M\left(E 0 ; 0_{2}^{+} \rightarrow\right.$ $\left.0_{1}^{+}\right)=2.1 e \mathrm{fm}^{2}$ as compared to experiment, 8.0(11) W.u. and $5.4(2) \mathrm{efm}^{2} 32$, respectively. Estimates of the latter include $6.53 \mathrm{efm}^{2}$ [8], 3(1) $e \mathrm{fm}^{2}$ [12], and $6.7 e \mathrm{fm}^{2}$ [9].

Comparison to ab initio results. - A close similarity is observed when the NCSpM wavefunctions of the g.st. rotational band are compared to ab initio results for the same $\hbar \Omega=18$ and $N_{\max }=6$ model space (Fig. $3 \mathrm{p}$ ). This space appears to be reasonable for these states for both models. In particular, we compare to wavefunctions obtained in the symmetry-adapted no-core shell model (SA-NCSM) 40 with bare JISP16 realistic interaction [41. The SA-NCSM utilizes an SU(3)-coupled basis, which yields conventional NCSM wavefunctions [23], but realized in terms of the $(\lambda \mu)$ deformation labels. The close agreement shows that among all possible configurations present in the SA-NCSM, only the states of the (04) and then (12) symplectic slices appear dominant. And if the SA-NCSM model space is reduced to only the spin components used in this study, $S_{\mathrm{p}} S_{\mathrm{n}} S=000,011$, and 101, NCSpM observables as g.st. matter rms radius and $Q_{2_{1}^{+}}$reproduce the ab initio counterparts as much as $80-90 \%$ and $70-90 \%$, respectively, for the same $\hbar \Omega$ and $N_{\max }=2,4$ and 6 . This suggests that the interaction used in NCSpM has effectively captured a good portion 
of the underlying physics of the realistic interaction important to the low-energy nuclear dynamics.

For the Hoyle state and its rotational band, larger spaces are needed, e.g., nonnegligible configurations extend to $N_{\max }=18$ (Fig. 3 a for the $0^{+}$state), which is within a reach of next-generation $a b$ initio NCSM models. For comparison, recent $a b$ initio $N_{\max }=8 \mathrm{NCSM}$ calculations, while achieving a remarkable reproduction of the g.st. rotational band, yield the second $0^{+}$and $2^{+}$ states around $13 \mathrm{MeV}$ and $15 \mathrm{MeV}$, respectively [11, thus believed not to be associated with the Hoyle state but with higher-lying states of that spin-parity. Indeed, consistent with $a b$ initio observations, the NCSpM outcome demonstrates a large sensitivity of the energy of the Hoyle state and its $2^{+}$excitation on the model space (Fig. 4 a). Finally, the additional degree of freedom associated with the $\gamma$ model parameter is in fact substantially limited by $0_{2}^{+}$and $0_{3}^{+}$and there is only a small window of reasonable $\gamma$ values (Fig. $4 \mathrm{~b}$ ), where observables are also found in agreement with experiment: for $\gamma$ from $10^{-2}$ to $10^{-5}$, e.g., $0_{\text {g.st. }}^{+}$and $0_{2}^{+} \mathrm{rms}$ radii increase 1.4 times, as well as $B\left(E 2 ; 2_{1}^{+} \rightarrow 0_{\text {g.st. }}^{+}\right)$and $Q_{2_{1}^{+}}$increase four times.

In short, we carried forward a no-core shell-model study with a many-nucleon interaction to further unveil the underlying physics behind various phenomena important to the low-energy nuclear dynamics of ${ }^{12} \mathrm{C}$. We showed, for the first time, how both collective states and states suggested to have cluster-like substructures emerge out of a fully microscopic, shell-model framework, thereby providing a novel and essential perspective on the controversial Hoyle state.

We thank Pieter Maris, James P. Vary, David J. Rowe, Catherine M. Diebel, and Moshe Gai for useful discussions. This work was supported by the U.S. NSF (OCI0904874), the U.S. DOE (de-sc0005248 \& DE-FG0295ER-40934), and the SURA. ACD acknowledges support by the U.S. NSF (grant 1004822) through the REU Site in Dept. of Physics \& Astronomy at LSU. We acknowledge LONI for providing HPC resources.

[1] H. O. U. Fynbo et al., Nature 433, 136 (2005).

[2] M. Freer et al., Phys. Rev. C 76, 034320 (2007); M. Freer et al. ibid. 80, 041303 (2009).

[3] S. Hyldegaard et al., Phys. Rev. C 81, 024303 (2010).

[4] M. Itoh et al., Phys. Rev. C 84, 054308 (2011).

[5] W. R. Zimmerman, N. E. Destefano, M. Freer, M. Gai, and F. D. Smit, Phys. Rev. C 84, 027304 (2011).

[6] Ad. R. Raduta et al., Phys. Letts. B 705, 65 (2011).

[7] W. R. Zimmerman et al., Phys. Rev. Lett. 110, 152502 (2013).

[8] M. Chernykh, H. Feldmeier, T. Neff, P. von NeumannCosel, and A. Richter, Phys. Rev. Lett. 98, 032501 (2007).

[9] D. T. Khoa, D. C. Cuonga, Y. Kanada-En'yo, Phys. Letts. B 695, 469 (2011).
[10] A. S. Umar, J. A. Maruhn, N. Itagaki, and V. E. Oberacker, Phys. Rev. Lett.104, 212503 (2010).

[11] R. Roth, J. Langhammer, A. Calci, S. Binder, and P. Navratil, Phys. Rev. Lett. 107, 072501 (2011).

[12] E. Epelbaum, H. Krebs, D. Lee, and Ulf-G. Meissner, Phys. Rev. Lett. 106, 192501 (2011); E. Epelbaum et al., Phys. Rev. Lett. 109, 252501 (2012).

[13] P. J. Ellis and T. Engeland, Nucl. Phys. A144, 161 (1970); T. Engeland and P. J. Ellis, Nucl. Phys. A181, 368 (1972); Y. Suzuki and K. T. Hecht, Nucl. Phys. A455, 315 (1986).

[14] J. P. Elliott, Proc. Roy. Sot. A245, 128 (1958); ibid. A245, 562 (1958); J. P. Elliott and M. Harvey, ibid. A272, 557 (1962).

[15] A. Bohr and B. R. Mottelson, Nuclear Structure, Benjamin, New York, Vol. 1 (1969) \& Vol. 2 (1974); B. R. Mottelson, Nobel Lectures, Physics 1971-1980, World Scientific Publishing Co., Singapore (1992).

[16] K. D. Launey, T. Dytrych, J. P. Draayer, G. K. Tobin, M. C. Ferriss, D. Langr, A. C. Dreyfuss, P. Maris, J. P. Vary, and C. Bahri, Proceedings of the 5th International Conference on "Fission and properties of neutron-rich nuclei", ICFN5, World Scientific Publishing Co., to be published (2013).

[17] G. Rosensteel and D. J. Rowe, Phys. Rev. Lett. 38, 10 (1977).

[18] T. Dytrych, K. D. Sviratcheva, C. Bahri, J. P. Draayer, and J. P. Vary, Phys. Rev. Lett. 98, 162503 (2007).

[19] G. Rosensteel and D. J. Rowe, Ann. Phys. 126, 343 (1980).

[20] J. P. Draayer, K. J. Weeks and G. Rosensteel, Nucl. Phys. A413,215 (1984).

[21] C. Bahri and D. J. Rowe, Nucl. Phys. A 662, 125 (2000).

$[22]$ T. Dytrych, K. D. Sviratcheva, J. P. Draayer, C. Bahri, and J. P. Vary, J. Phys. G: Nucl. Part. Phys. 35, 123101 (2008).

[23] P. Navrátil, J. P. Vary, and B. R. Barrett, Phys. Rev. Lett. 84, 5728 (2000); B.R. Barrett, P. Navrátil and J.P. Vary, Prog. Part. Nucl. Phys. 69, 131 (2013); P. Maris, J. P. Vary and P. Navrátil, Phys. Rev. C 87, 014327 (2013).

[24] G. Rosensteel and D. J. Rowe, Ann. Phys. N.Y. 104, 134 (1977); Y. Leschber and J. P. Draayer, Phys. Letts. B 190, 1 (1987);O. Castaños, J. P. Draayer, and Y. Leschber, Z. Phys 329, 33 (1988).

[25] M. Harvey, Adv. Nucl. Phys. 1, 62 (1968).

[26] G. Rosensteel and J. P. Draayer, Nucl. Phys. A436, 445 (1985).

[27] O. Castaños and J. P. Draayer, Nucl. Phys. A491, 349 (1989).

[28] D. R. Peterson and K. T. Hecht, Nucl. Phys. A344, 361 (1980).

[29] R. Le Blanc, J. Carvalho, M. Vassanji, and D.J. Rowe, Nucl. Phys. A452, 263 (1986).

[30] D. J. Rowe, Phys. Rev. 162, 866 (1967).

[31] D. J. Rowe, G. Thiamova, and J. L. Wood, Phys. Rev. Lett. 97, 202501 (2006).

[32] F. Ajzenberg-Selove and J.H. Kelley, Nucl. Phys. A506, 1 (1990).

[33] M. Freer et al., Phys. Rev. C 83 (2011).

[34] Y. Kanada-En'yo, Phys. Rev. Lett. 81, 5291 (1998).

[35] I. Tanihata et al., Phys. Rev. Lett. 55, 2676 (1985).

[36] A. N. Danilov, T. L. Belyaeva, A. S. Demyanova, S. A. Goncharov, and A. A Ogloblin, Phys. Rev. C 80, 054603 (2009).

[37] A. A Ogloblin, T. L. Belyaeva, A. N. Danilov, A. S. De- 
myanova, and S. A. Goncharov, Eur. Phys. J. A 49, 46 (2013).

[38] Y. Funaki, A. Tohsaki, H. Horiuchi, P. Schuck, and G. Ropke, Phys. Rev. C 67, 051306 (2003).

[39] T. Yamada and P. Schuck, Eur. Phys. J. A 26, 185 (2005).

[40] T. Dytrych et al., submitted to Phys. Rev. Lett. (2013);
J. P. Draayer, T. Dytrych, K. D. Launey and D. Langr, Prog. Part. Nucl. Phys. 67, 516 (2012).

[41] A. M. Shirokov, J. P. Vary, A. I. Mazur, and T. A. Weber, Phys. Letts. B 644, 33 (2007). 